\title{
Knowledge of University Athletes about Knowing and Monitoring of Vital Signs of Blood Pressure (BP), Heart Rate (HR) and Body Mass Index (BMI) as Preventive Strategy in Reducing Early and Unsuccessful Ageing Cased at Njala Campus
}

\section{Bebeley Samuel Joseph, Laggao Sam Augustine and Tucker Henry Joe}

\begin{abstract}
Aim: To assess the preventive strategy in reducing early and unsuccessful ageing among University Athletes using Njala Campus as a case study, with the significance to measure and evaluate the knowledge of University Athlete about knowing and monitoring of vital signs of blood pressure [BP], heart rate [HR] and body mass index [BMI] as preventive strategy in reducing early and unsuccessful ageing. Method: The modified health risk behavioural survey questionnaire [MHRBSQ] was adopted for testing the parameters. The respondents interviewed were mainly undergraduate athletes with an aggregate of one hundred and fifty [n=150]. However, 55 with $37 \%$ were female and 95 with $63 \%$ were male, selected using the systematic random sampling. Also sampled were four Schools: 25 with $16.6 \%$ female and 40 with $26.6 \%$ male from the School of Agriculture \& Environmental Science; 30 with $20 \%$ female and 55 with $37 \%$ male from the School of Education \& Technology, from level [100-200] and from level [300-400], within the age range of [18-30 year]. Results: Analysis of findings from knowing and monitoring of vital signs of blood pressure $[\mathrm{BP}]$, heart rate $[\mathrm{HR}]$ and body mass index $[\mathrm{BMI}]$, show a holistic significant difference in all three variables [t-values of 4.666, 4.711 and $3.368 \mathrm{p}<0.05]$.Conclusion: Conclusively therefore, the findings show that majority of the University Athletes were experienced holistically in their knowledge about preventive strategy in reducing early and unsuccessful ageing with respect to the evaluated variables. It was recommended that University Athletes be given thoroughly supervised seminars, workshops and screening prior to any intercollegiate competitions held, with special reference to knowledge of vital signs.
\end{abstract}

\author{
Bebeley Samuel Joseph \\ Lecturer \\ Department of Human Kinetics and Health Education \\ Njala University, Sierra Leone \\ E-mail: bsaj2004@hotmail.co.uk \\ Laggao Sam Augustine \\ HOD Human Kinetics and Health Education \\ Njala University, Sierra Leone \\ Tucker Henry Joe \\ Lecturer \\ Department of Human Kinetics and Health Education \\ Njala University, Sierra Leone
}

Key Words: Ageing, Athlete, Blood Pressure, Body Mass Index, Health, Physical Fitness

DOI: $10.18376 /$ jesp/2017/v13/i1/111270

\section{Introduction}

Vital signs [shortened as vitals] are used to measure the body's basic functions. These measurements are taken to help assess the general physical health of a person, give clues to possible diseases, and show progress toward recovery. The normal ranges for a person's vital signs vary with age, weight, gender, and overall health, National Early Warning Score Development and 


\section{Journal of Exercise Science \& Physiotherapy, Vol. 13, No. 1, 2017 \\ ISSN: 0973-2020 (Print) I2OR Impact Factor $=5.23$ UGC Approved [no.20489] ISSN: 2454-6089 \\ (Online)}

Implementation Group (2012). There are four primary vital signs: body temperature, blood pressure, pulse (heart rate), and breathing rate (respiratory rate), often notated as BT, BP, HR, and RR. However, depending on the clinical setting, the vital signs may include other measurements called fifth or sixth vital sign such as the body mass index shortened as BMI. Vital signs are recorded using the LOINC internationally accepted standard coding system, National Institute for Health and Clinical Excellence (2007).Early warning scores have been proposed that combine the individual values of vital signs into a single score. This was done in recognition that deteriorating vital signs often precede cardiac arrest and/or admission to the intensive care unit. Used appropriately, a rapid response team can assess and treat a deteriorating patient and prevent adverse outcomes, Acute Care Toolkit (2013).

Wellness is generally used to mean a healthy balance of the mind, body and spirit that results in an overall feeling of well-being. It has been used in the context of alternative medicine since Halbert, L. Dunn, M.D., began using the phrase high-level wellness in the 1950s. The modern concept of wellness did not, however, become popular until the 1970s, Zimmer, B. (2010).Halbert, L. and Dunn, M. D., began using the phrase high-level wellness in the 1950 s, based on a series of lectures at a Unitarian Universalist Church in Arlington, Virginia, in the United States. Dunn (196, p. 4) defined wellness as "an integrated method of functioning which is oriented toward maximizing the potential of which the individual is capable. It requires that the individual maintain a continuum of balance and purposeful direction within the environment where he is functioning." He also stated that, "wellness is a direction in progress toward an ever-higher potential of functioning" (p. 6). Dunn also described wellness as health being, "much more than the absence of disease remains a cornerstone concept of wellness today." (Dunn, 787, p 7) Dunn saw wellness as hierarchical: there were lower levels of wellness and higher ones, and the aim was to move everyone up from where they started to high-level wellness (Dunn, 789, p 8), Neilson, E. A. (1988).

Health is that balanced condition of the living organism in which the integral, harmonious performance of the vital functions tends to the preservation of the organism and the normal development of the individual, World Health Organization (2011). Physical fitness is a measure of the individual body's ability and capability to function efficiently and effectively during work and leisure times, with the tendency of resisting hypokinetic diseases, and to meet unforeseen situations, Colfer, G. R. (2004).

Blood pressure (BP) is the pressure exerted by circulating blood upon the walls of blood vessels. When used without further specification, "blood pressure" usually refers to the arterial pressure in the systemic circulation. It is usually measured at a person's upper arm. Blood pressure is usually expressed in terms of the systolic (maximum) pressure over diastolic (minimum) pressure and is measured in millimeters of mercury $(\mathrm{mmHg})$. It is one of the vital signs along with respiratory rate, heart rate, oxygen saturation and body temperature. Normal resting blood pressure in an adult is approximately 120/80 mmHg, American Heart Association (2011).Blood pressure varies depending on situation, activity, and disease states. It is regulated by the nervous and endocrine systems. Blood pressure that is low due to a disease state is called hypotension, and pressure that is consistently high is hypertension. Both have many causes, which can range from mild to severe. Both may be of sudden onset or of long duration. Long-term hypertension is a risk factor for many diseases, including kidney failure, heart disease and stroke. Long-term hypertension is more common than long-term hypotension in Western countries. Long-term hypertension often goes undetected because of infrequent monitoring and the absence of symptoms, Mayo Clinic staff (2009).In the UK, clinic blood pressures are usually categorized into three groups; low (90/60 or lower), normal (between 90/60 and 139/80), and high (140/90 or higher), National Heart Lung and Blood Institute(2008). Systolic and diastolic arterial blood pressures are not static but undergo 


\section{Journal of Exercise Science \& Physiotherapy, Vol. 13, No. 1, 2017 \\ ISSN: 0973-2020 (Print) $\quad$ I $_{2}$ OR Impact Factor $=5.23 \quad$ UGC Approved [no.20489] ISSN: 2454-6089 \\ (Online)}

natural variations from one heartbeat to another and throughout the day (in a circadian rhythm). They also change in response to stress, nutritional factors, drugs, disease, exercise, and momentarily from standing up. Sometimes the variations are large. Hypertension refers to arterial pressure being abnormally high, as opposed to hypotension, when it is abnormally low. Along with body temperature, respiratory rate, and pulse rate, blood pressure is one of the four main vital signs routinely monitored by medical professionals and healthcare providers, Deakin, C. D. et al.(2000). Heart rate (HR), or heart pulse, is the speed of the heartbeat measured by the number of poundings of the heart per unit of time - typically beats per minute (bpm). The heart rate can vary according to the body's physical needs, including the need to absorb oxygen and excrete carbon dioxide. Activities that can provoke change include physical exercise, sleep, anxiety, stress, illness, ingesting, and drugs. The normal resting adult human heart rate ranges from 60-100 (bpm), "Target Heart Rates - AHA" (2014). Tachycardia is a fast heart rate, defined as above 100 (bpm) at rest, "Tachycardia Fast Heart Rate" (2013). Bradycardia is a slow heart rate, defined as below 60 (bpm) at rest. During sleep a slow heartbeat with rates around 40-50 (bpm) is common and is considered normal. When the heart is not beating in a regular pattern, this is referred to as an arrhythmia. These abnormalities of heart rate sometimes indicate disease, "Tachycardia Fast Heart Rate" (2013). While heart rhythm is regulated entirely by the Sino atrial node under normal conditions, heart rate is regulated by sympathetic and parasympathetic input to the Sino atrial node. The accelerants nerve provides sympathetic input to the heart by releasing norepinephrine onto the cells of the Sino atrial node, and the vagus nerve provides parasympathetic input to the heart by releasing acetylcholine onto Sino atrial node (SAN) cells. Therefore, stimulation of the accelerants nerve increases heart rate, while stimulation of the vagus nerve decreases it, Schmidt-Nielsen and Knut (1997).Due to individuals having a constant blood volume, one of the physiological ways to deliver more oxygen to an organ is to increase heart rate to permit blood to pass by the organ more often, "Tachycardia Fast Heart Rate" (2013). Normal resting heart rates range from 60-100 (bpm). Bradycardia is defined as a resting heart rate below 60 (bpm). However, heart rate(s) from 50 to 60 (bpm) are common among healthy people and do not necessarily require special attention. Tachycardia is defined as a resting heart rate above $100(\mathrm{bpm})$, though persistent rest rates between 80-100 (bpm), mainly if they are present during sleep, may be signs of hyperthyroidism or anemia, "Tachycardia Fast Heart Rate" (2013). There are many ways in which the heart rate speeds up or slows down. Most involve stimulant-like endorphins and hormones being released in the brain, many of which are those that are 'forced'/'enticed' out by the ingestion and processing of drugs, Anderson, J. M. (1991).

Body mass index (BMI), or Quetelet index, is a value derived from the mass (weight) and height of an individual. The BMI is defined as the body mass divided by the square of the body height, and is universally expressed in units of $\mathrm{kg} / \mathrm{m}^{2}$, resulting from weight in kilograms and height in meters, World Health Organization BMI Classification (2006) or chart which displays BMI as a function of mass and height using contour lines or colors for different BMI categories, and may use two different units of measurement, Eknoyan and Garabed (2007). The BMI is an attempt to quantify the amount of tissue mass (muscle, fat, and bone) in an individual, and then categorize that person as underweight, normal weight, overweight, or obese based on that value. However, there is some debate about where on the BMI scale the dividing lines between categories should be placed. Commonly accepted BMI ranges are underweight: 18.5 , normal weight: 18.5 to 25 , overweight: 25 to 30, obese: over $30\left(\mathrm{~kg} / \mathrm{m}^{2}\right)$, Malcolm, K., Dr. (2015). There are criticisms of using the BMI to define obesity in individuals. One is that the BMI was designed for population studies, not individuals. Another is that body fat percentage (BFP) is a more reliable indicator of obesity than BMI: very muscular, lean (low body fat) individuals can be classified as obese using BMI, but are 


\section{Journal of Exercise Science \& Physiotherapy, Vol. 13, No. 1, 2017 \\ ISSN: 0973-2020 (Print) $\quad$ I $_{2}$ OR Impact Factor $=5.23 \quad$ UGC Approved [no.20489] ISSN: 2454-6089 \\ (Online)}

classified as having a normal weight using BFP. An even simpler alternative to the BMI is to define obese individuals as those whose waist circumference is greater than $50 \%$ of their height, indicating excess intra-abdominal fat, Jeremy Singer-Vine (2009).

The index was devised by Adolphe Quetelet (1830-1850) during which time he developed what he called "social physics", Eknoyan and Garabed (2007). The modern term "body mass index" (BMI) for the ratio of weight to squared height owes its popularity to a paper published in the July 1972 edition of the Journal of Chronic Diseases by Ancel Keys. This found the BMI to be the best proxy for body fat percentage among ratios of weight and height, Keys, A. et al. (1972). The interest in an index that measures body fat came with increasing obesity in prosperous Western societies. Keys explicitly cited BMI as appropriate for population studies and inappropriate for individual evaluation. Nevertheless, due to its simplicity, it has come to be widely used for preliminary diagnosis, National Heart, Lung and Blood Institute (2014).

Collegiate athletes by definition are athletes that are engaged in organized games and sports competition sponsored by individual educational institutions. Gerdy, R. (2000). Collegiate athletes that give positive reason to the contraindications posed by behavioral risk factors, Muffuli, et al. (2003) that progresses unsuccessful ageing is referred to as primary prevention knowledge. However, successful ageing refers to physical, mental and social well-being in older age. The concept of successful aging can be traced back to the 1950s, and was popularized in the 1980s. It reflects changing view on aging in Western countries, where a stigma associated with old age(see ageism) has led to considering older people as a burden on society. Consequently, in the past most of the scientists have been focusing on negative aspects of aging or preventing the decline of youth, Rowe, J. et al. (1997) and Fries, J. F. (2002). Research on successful aging, however, acknowledges the fact that there is a growing number of older adults functioning at a high level and contributing to the society. Scientists working in this area seek to define what differentiates successful from usual aging in order to design effective strategies and medical interventions to protect health and well-being from aging, Rowe, J. et al. (1997); Cantoni, G. (1998); WHO (2003); Peel, N. M. et al. (2005); Phelan, E. A. (2002) and Lupien, S. J. (2004). Researchers in ageing studies are critical of the very term 'successful ageing' as it implies failure on the part of those who do not meet arbitrary criteria derived from neoliberal and/or biomedical definitions, Katz, S. (2015).

This study only looked at the assessment ofUniversity Athletes knowledge about the knowing and monitoring of vital signs of blood pressure [BP], heart rate [HR] and body mass index [BMI], as preventive strategy in reducing early and unsuccessful ageing, ranked through 100-to-200 and 300to-400 levels, thereby pointing outcomparatively the significant differences between the dependent variables regarding blood pressure $[\mathrm{BP}]$, heart rate $[\mathrm{HR}]$ and body mass index $[\mathrm{BMI}]$ cased at Njala Campus.

\section{Materials and Methods}

Respondents interviewed were mainly undergraduates' athletes with an aggregate of one hundred and fifty [n=150]. However, 55 with $37 \%$ were female and 95 with $63 \%$ were male; 30 with $20 \%$ female Christians and 40 with $27 \%$ male Christians; 25 with $16.6 \%$ female Muslims and 55 with $36.6 \%$ male Muslims; 4 with 3\% married female and 6 with $4 \%$ married male; 51 with $34 \%$ female single and 89 with $59 \%$ male single; 35 with $23.3 \%$ South-East female and 65 with $43.3 \%$ SouthEast male; 20 with $13 \%$ North-West female and 30 with $20 \%$ North-West male, were selected using the systematic random sampling. Also sampled were four Schools: 25 with $16.6 \%$ female and 40 with $26.6 \%$ male from the School of Agriculture \& Environmental Science; 30 with $20 \%$ female and 55 with $37 \%$ male from the School of Education \& Technology; 45 with $30 \%$ female and 80 with 53\% male from level [100-200]; 10 with 7\% female and 15 with $10 \%$ male from level [300- 


\section{ISSN: 0973-2020 (Print) I2OR Impact Factor $=5.23$ UGC Approved [no.20489] ISSN: 2454-6089 \\ (Online)}

400]; 30 with $20 \%$ female and 60 with $40 \%$ male were within the age range of [18-25]; 25 with $17 \%$ female and 35 with $23 \%$ male were within the age range of [26-30+] in years.

Instrument for Measuring Parameters

The descriptive survey research design was used for the research. The dependent variables tested were: knowing and monitoring of vital signs of blood pressure [BP], heart rate [HR] and body mass index [BMI]. The modified health risk behavioural survey questionnaire [MHRBSQ] was adopted as the research instrument for testing the parameters that was formally worked on by Bebeley, $S . J$. (2016). The questionnaire was supported with Section-One demographic data and Section-Two variable data. Senior colleagues in the Department of Human Kinetics and Health Education, Njala University and College of Physical Education and Sport Training, Shanghai University of Sport, validated the questionnaire as research instrument which was pre-tested on an aggregate of 50 Polytech Athletes from Kenema using the test retest method, thereby producing a high reliability of 0.99 as referenced in tables $1-2$ below.

Test Procedures

Respondents interviewed were mainly undergraduate University Athletes with an aggregate of one hundred and fifty [n=150] rankedthrough 100-to-200 and 300-to-400 levels and Schooledthrough Agriculture-to-Environmental Science and Education-to-Technology, Njala Campus. The respondents were interviewed by the researchers helped by some academic staff members of the Department of Human Kinetics and Health Education adopting the face-to-face logic based on the dependent variables, before the Campus Sport Complex prior to training session adhering strictly to the dependent variables in their response. The individual responses were compiled for statistical analyses.

Statistical Analysis

The standard deviation, mean, inferential statistics of Dependent t-test [t], percentage and frequency distribution tables, were adopted for analyzing the data obtained from University Athletes using Njala Campus as case study about their knowledge of preventive strategy in decreasing early ageing through a modified health risk behavioural survey questionnaire [MHRBSQ] as research instrument for testing the variables, which was formally used by Bebeley, et al. (2016), objected towards plausible differences on the views of University Athletes knowledge about preventive strategy in reducing early and unsuccessful ageing. The results were evaluated at level of significance $\mathrm{p}<0.05$.

\section{Results}

Table 1. Reliability Test-retest Demographic Data of Respondents according to Gender $[\mathrm{n}=50]$

\begin{tabular}{|c|c|c|c|c|c|c|c|c|c|c|c|c|}
\hline \multirow[t]{3}{*}{ Gender } & \multicolumn{4}{|c|}{ Age } & \multicolumn{2}{|c|}{$\mathbf{P}^{2}$} & \multicolumn{2}{|c|}{$\overline{\mathbf{Q}^{2}}$} & \multirow{2}{*}{\multicolumn{2}{|c|}{$\begin{array}{c}\mathbf{T i} \\
\mathbf{R}_{\mathbf{1}} \sum \\
\mathbf{R}_{\mathbf{2}}\end{array}$}} & \multirow{2}{*}{\multicolumn{2}{|c|}{$\frac{\mathbf{T i}^{\mathbf{2}}}{\mathbf{R}_{1} \sum \mathbf{R}_{\mathbf{2}}}$}} \\
\hline & \multicolumn{2}{|c|}{$\begin{array}{c}\mathbf{P} \\
{[18-25]}\end{array}$} & \multicolumn{2}{|c|}{$\begin{array}{c}\mathbf{Q} \\
{[26-35+]}\end{array}$} & \multirow[t]{2}{*}{$\mathbf{R}_{1}$} & \multirow[t]{2}{*}{$\mathbf{R}_{2}$} & \multirow[t]{2}{*}{$\mathbf{R}_{1}$} & \multirow[t]{2}{*}{$\mathbf{R}_{2}$} & & & & \\
\hline & $\mathbf{R}_{1}$ & $\mathbf{R}_{2}$ & $\mathbf{R}_{1}$ & $\mathbf{R}_{2}$ & & & & & & $\mathbf{Q}$ & $\mathbf{P}$ & $\mathbf{Q}$ \\
\hline Mal & 25 & 24 & 10 & 11 & 625 & 576 & 100 & 121 & 49 & 21 & 2401 & 441 \\
\hline Female & 10 & 09 & 05 & 06 & 100 & 81 & 25 & 36 & 19 & 11 & 361 & 121 \\
\hline \multirow[t]{2}{*}[\mathrm{n}=2]{} & \multicolumn{2}{|c|}{$* \sum P=68$} & \multicolumn{2}{|c|}{$* \sum \mathbf{Q}=\mathbf{3 2}$} & $\begin{array}{l}* \sum \mathbf{P}^{2} \\
1382\end{array}$ & $=$ & $\begin{array}{l}* \sum Q^{2} \\
282\end{array}$ & $=$ & & $\mathrm{Ti}^{2}$ & 2762 & 562 \\
\hline & \multicolumn{2}{|c|}{$*\left[\sum P\right]^{2}=4624$} & \multicolumn{2}{|c|}{$*[\Sigma \mathbf{Q}]^{2}=\mathbf{1 0 2 4}$} & \multicolumn{8}{|c|}{$*$ Reliability $=0.99$} \\
\hline
\end{tabular}


Journal of Exercise Science \& Physiotherapy, Vol. 13, No. 1, 2017

ISSN: 0973-2020 (Print) I I OR Impact Factor $=5.23$ UGC Approved [no.20489]

(Online)

\begin{tabular}{|c|c|c|c|c|c|c|c|c|c|c|c|c|}
\hline \multirow{4}{*}{ Gender } & \multicolumn{4}{|c|}{ Marital Status } & \multicolumn{2}{|c|}{$\mathbf{P}^{2}$} & \multicolumn{2}{|c|}{$\mathbf{Q}^{2}$} & \multicolumn{2}{|c|}{$\mathbf{T i}$} & \multicolumn{2}{|c|}{$\mathbf{T i}^{2}$} \\
\hline & \multirow{2}{*}{\multicolumn{2}{|c|}{$\begin{array}{c}\mathbf{P} \\
\text { [Single] }\end{array}$}} & \multirow{2}{*}{\multicolumn{2}{|c|}{$\begin{array}{c}\mathbf{Q} \\
{[\text { Married] }}\end{array}$}} & \multirow{3}{*}{$\mathbf{R}_{1}$} & \multirow{3}{*}{$\mathbf{R}_{\mathbf{2}}$} & \multirow{3}{*}{$\mathbf{R}_{1}$} & \multirow{3}{*}{$\mathbf{R}_{2}$} & \multirow{2}{*}{\multicolumn{2}{|c|}{$\begin{array}{c}\mathbf{R}_{\mathbf{1}} \sum \\
\mathbf{R}_{\mathbf{2}}\end{array}$}} & \multirow{2}{*}{\multicolumn{2}{|c|}{$\mathbf{R}_{\mathbf{1}} \sum \mathbf{R}_{\mathbf{2}}$}} \\
\hline & & & & & & & & & & & & \\
\hline & $\mathbf{R}_{1}$ & $\mathbf{R}_{2}$ & $\mathbf{R}_{1}$ & $\mathbf{R}_{2}$ & & & & & & $\mathbf{Q}$ & $\mathbf{P}$ & $\mathbf{Q}$ \\
\hline Male & 30 & 29 & 08 & 09 & 900 & 841 & 64 & 81 & 59 & 17 & 3481 & 289 \\
\hline Fen & 10 & 09 & 02 & 03 & 100 & 81 & 04 & 09 & 19 & 05 & 361 & 25 \\
\hline \multirow{2}{*}[n=2]{} & \multicolumn{2}{|c|}{$* \sum P=78$} & \multicolumn{2}{|c|}{$* \sum \mathbf{Q}=\mathbf{2 2}$} & \multicolumn{2}{|c|}{$* \sum P^{2}=1922$} & \multicolumn{2}{|c|}{$* \sum Q^{2}=158$} & \multicolumn{2}{|c|}{$* \sum \mathbf{T i}^{2}$} & \multirow[t]{2}{*}{3842} & \multirow[t]{2}{*}{314} \\
\hline & $*\left[\sum\right]$ & 6084 & $*\left[\sum \mathbf{C}\right.$ & 484 & & & & & $=0$. & & & \\
\hline
\end{tabular}

$$
\begin{array}{ll}
\text { Religion Category } \\
\mathbf{P}
\end{array}
$$$$
\begin{array}{lll}
\mathbf{P}^{2} & \mathbf{Q}^{2}
\end{array}
$$$$
\mathbf{T i}^{2}
$$

ISSN: 2454-6089

Gender [Muslim] [Christia

\begin{tabular}{|c|c|c|c|c|c|c|c|c|c|c|c|c|}
\hline \multirow{3}{*}{ Gender } & \multicolumn{4}{|c|}{ Region Allocation } & \multicolumn{2}{|c|}{$\mathbf{P}^{2}$} & \multicolumn{2}{|c|}{$\mathbf{Q}^{2}$} & \multirow{2}{*}{\multicolumn{2}{|c|}{$\begin{array}{c}\mathbf{T i} \\
\mathbf{R}_{\mathbf{1}} \sum \\
\mathbf{R}_{\mathbf{2}}\end{array}$}} & \multirow{2}{*}{\multicolumn{2}{|c|}{$\begin{array}{c}\mathbf{T i}^{2} \\
\mathbf{R}_{\mathbf{1}} \sum \mathbf{R}_{\mathbf{2}}\end{array}$}} \\
\hline & \multicolumn{2}{|c|}{$\begin{array}{c}\mathbf{P} \\
{[\text { South/East] }}\end{array}$} & \multicolumn{2}{|c|}{$\begin{array}{c}\mathbf{Q} \\
{[\text { North/West }]}\end{array}$} & \multirow[t]{2}{*}{$\mathbf{R}_{1}$} & \multirow[t]{2}{*}{$\mathbf{R}_{\mathbf{2}}$} & \multirow[t]{2}{*}{$\mathbf{R}_{1}$} & \multirow[t]{2}{*}{$\mathbf{R}_{2}$} & & & & \\
\hline & $\mathbf{R}_{1}$ & $\mathbf{R}_{2}$ & $\mathbf{R}_{1}$ & $\mathbf{R}_{2}$ & & & & & $\mathbf{P}$ & $\mathbf{Q}$ & $\mathbf{P}$ & $\mathbf{Q}$ \\
\hline Male & 22 & 23 & 15 & 14 & 484 & 529 & 225 & 196 & 45 & 29 & 2025 & 841 \\
\hline Female & 08 & 09 & 05 & 04 & 64 & 81 & 25 & 16 & 17 & 09 & 289 & 81 \\
\hline$[\mathbf{n}=2]$ & $\begin{array}{r}* \sum \\
* \Gamma \Gamma !\end{array}$ & 62 & $* \Gamma \Gamma$ & 1444 & & & & $2=$ & $* \sum$ & $\mathbf{T i}^{2}$ & 2314 & 922 \\
\hline
\end{tabular}

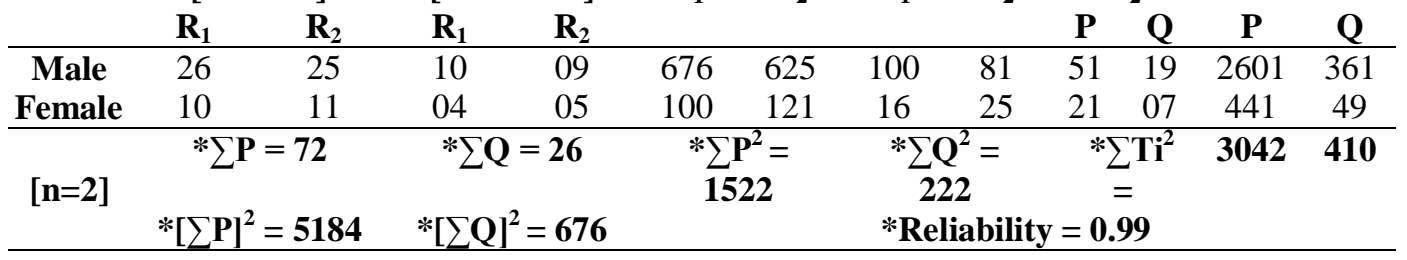

\begin{tabular}{ll}
\hline Gender & School of Studies \\
& $\mathbf{P}$
\end{tabular}

\begin{tabular}{|c|c|c|c|c|c|c|c|c|c|c|c|c|}
\hline & $R_{1}$ & $\mathbf{R}_{2}$ & $\mathbf{R}_{1}$ & $\mathbf{R}_{2}$ & & & & & $\mathbf{P}$ & Q & $\mathbf{P}$ & $\mathbf{Q}$ \\
\hline Male & 24 & 25 & 12 & 11 & 576 & 625 & 144 & 121 & 49 & 23 & 2401 & 529 \\
\hline Female & 06 & 07 & 08 & 07 & 36 & 49 & 64 & 49 & 13 & 15 & 169 & 225 \\
\hline$[\mathrm{n}=2]$ & $\begin{array}{r}* 2 \\
*[\Gamma\end{array}$ & 3844 & $\begin{array}{r}* \Sigma \\
* \Gamma \Gamma 0\end{array}$ & $\begin{array}{l}=38 \\
1444\end{array}$ & & & & $\begin{array}{l}2= \\
3 \\
\end{array}$ & $=0$. & $\mathbf{T i}^{2}$ & 2570 & 754 \\
\hline
\end{tabular}

$\left[\begin{array}{lllllll}\text { [Edu/Tech] } & \text { [Ag./Env Sc.] } & \mathbf{R}_{1} & \mathbf{R}_{2} & \mathbf{R}_{1} & \mathbf{R}_{2} & \mathbf{R}_{2}\end{array}\right.$

\begin{tabular}{|c|c|c|c|c|c|c|c|c|c|c|c|c|}
\hline \multirow{3}{*}{ Gender } & \multicolumn{4}{|c|}{ Level of Academe } & \multicolumn{2}{|c|}{$\mathbf{P}^{2}$} & \multicolumn{2}{|c|}{$\mathbf{Q}^{2}$} & \multirow{2}{*}{\multicolumn{2}{|c|}{$\begin{array}{c}\mathbf{T i} \\
\mathbf{R}_{\mathbf{1}} \sum \\
\mathbf{R}_{\mathbf{2}}\end{array}$}} & \multirow{2}{*}{\multicolumn{2}{|c|}{$\begin{array}{c}\mathbf{T i}^{\mathbf{2}} \\
\mathbf{R}_{1} \sum^{2} \mathbf{R}_{2}\end{array}$}} \\
\hline & \multicolumn{2}{|c|}{$\begin{array}{c}P \\
{\left[\mathbf{L v}_{1}-\mathbf{L v}_{2}\right]}\end{array}$} & \multicolumn{2}{|c|}{$\begin{array}{c}\mathbf{Q} \\
{\left[\mathbf{L} v_{3}-\mathbf{L} v_{4}\right]}\end{array}$} & \multirow[t]{2}{*}{$\mathbf{R}_{\mathbf{1}}$} & \multirow[t]{2}{*}{$\mathbf{R}_{2}$} & \multirow[t]{2}{*}{$\mathbf{R}_{1}$} & \multirow[t]{2}{*}{$\mathbf{R}_{2}$} & & & & \\
\hline & $\mathbf{R}_{1}$ & $\mathbf{R}_{\mathbf{2}}$ & $\mathbf{R}_{\mathbf{1}}$ & $\mathbf{R}_{\mathbf{2}}$ & & & & & $\mathbf{P}$ & $\mathbf{Q}$ & $\mathbf{P}$ & $\mathbf{Q}$ \\
\hline Male & 28 & 29 & 10 & 09 & 784 & 841 & 100 & 81 & 57 & 19 & 3249 & 361 \\
\hline Female & 10 & 11 & 02 & 01 & 100 & 121 & 04 & 01 & 21 & 03 & 441 & 09 \\
\hline
\end{tabular}


Journal of Exercise Science \& Physiotherapy, Vol. 13, No. 1, 2017

ISSN: 0973-2020 (Print) $\quad \mathrm{I}_{2}$ OR Impact Factor $=5.23 \quad$ UGC Approved [no.20489] ISSN: 2454-6089 (Online)

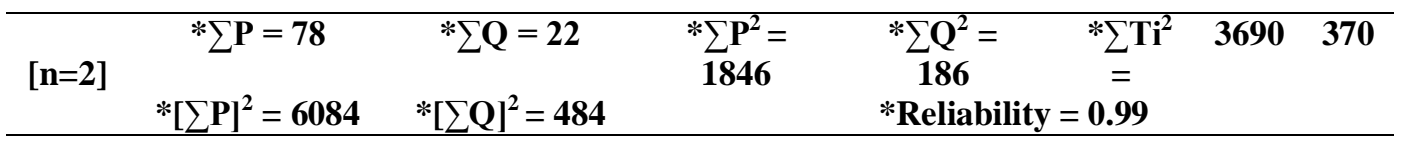

Table 2. Reliability Test-retest of Polytechnic Athletes' knowledge about knowing\& monitoring of vital signs as primary preventive strategy in reducing early and unsuccessful

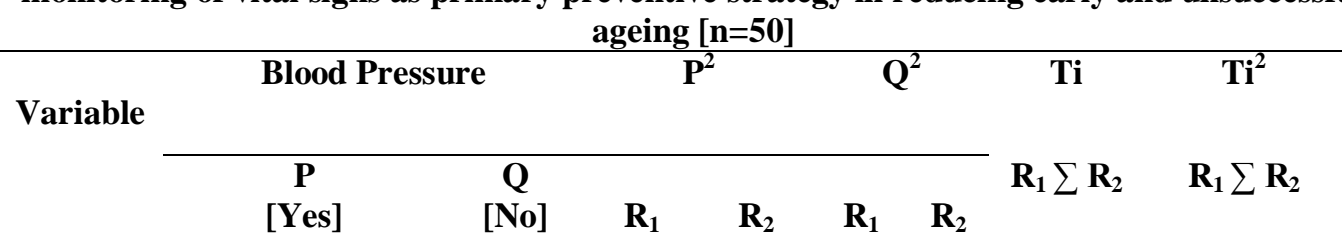

Variable

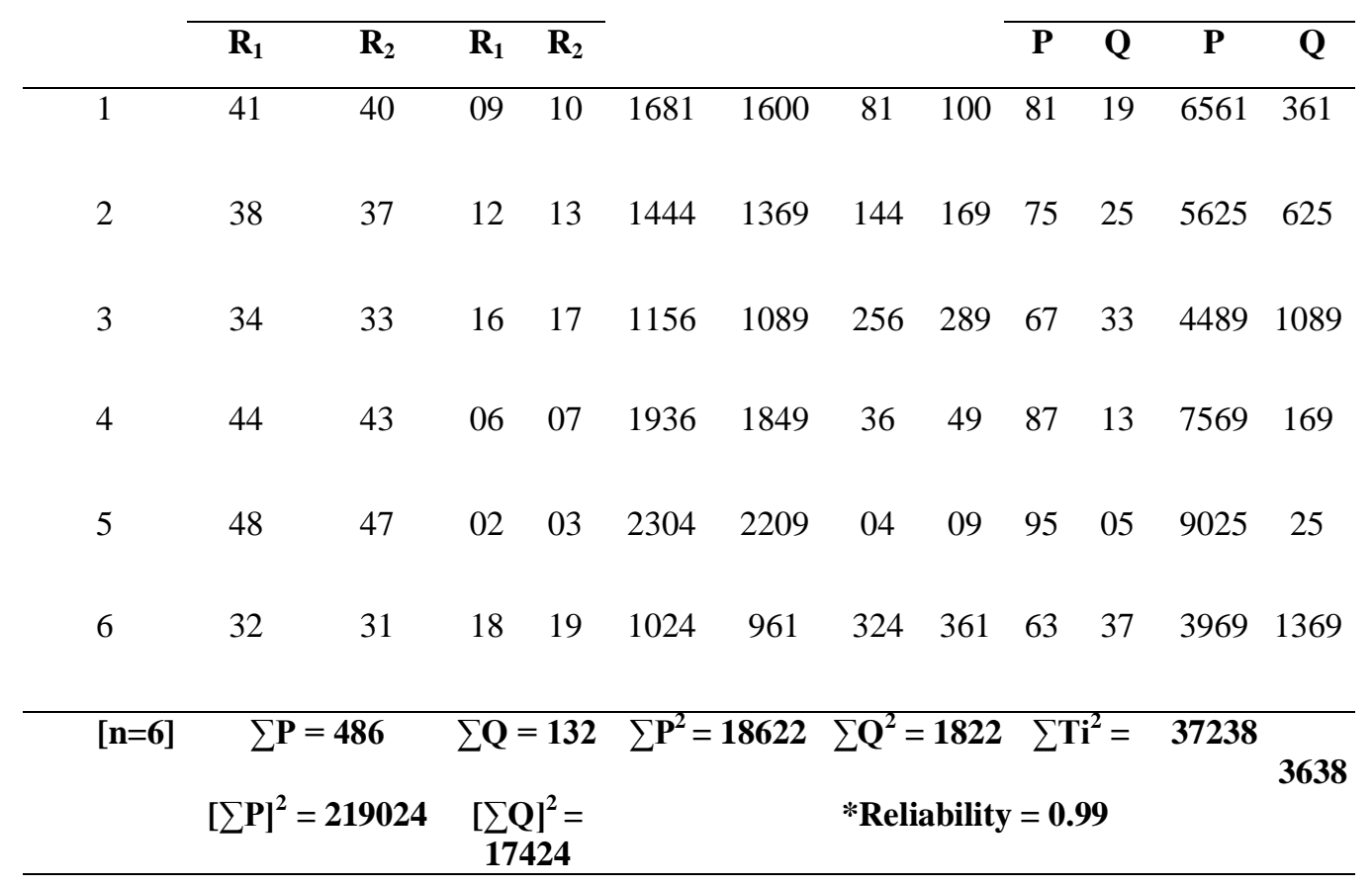

\begin{tabular}{|c|c|c|c|c|c|c|c|c|c|}
\hline \multirow{3}{*}{ Variable } & \multicolumn{3}{|c|}{ Heart Rate } & \multicolumn{2}{|c|}{$\mathbf{P}^{2}$} & \multicolumn{2}{|c|}{$\mathbf{Q}^{2}$} & \multirow{2}{*}{$\begin{array}{c}\mathbf{T i} \\
\mathbf{R}_{\mathbf{1}} \sum \mathbf{R}_{\mathbf{2}}\end{array}$} & \multirow{2}{*}{$\begin{array}{c}\mathbf{T i}^{\mathbf{2}} \\
\mathbf{R}_{\mathbf{1}} \sum \mathbf{R}_{\mathbf{2}}\end{array}$} \\
\hline & & & $\begin{array}{c}\mathbf{Q} \\
{[\mathrm{No}]}\end{array}$ & \multirow[t]{2}{*}{$\mathbf{R}_{1}$} & \multirow[t]{2}{*}{$\mathbf{R}_{2}$} & \multirow[t]{2}{*}{$\mathbf{R}_{1}$} & \multirow[t]{2}{*}{$\mathbf{R}_{2}$} & & \\
\hline & $\mathbf{R}_{1}$ & $\mathbf{R}_{2}$ & $\begin{array}{ll}\mathbf{R}_{1} & \mathbf{R}_{2}\end{array}$ & & & & & $\mathbf{P}$ & $\mathbf{Q}$ \\
\hline 1 & 39 & 38 & $11 \quad 12$ & 1521 & 1444 & 121 & 144 & $77 \quad 23$ & $5929 \quad 529$ \\
\hline
\end{tabular}


Journal of Exercise Science \& Physiotherapy, Vol. 13, No. 1, 2017

ISSN: 0973-2020 (Print) $\quad \mathrm{I}_{2}$ OR Impact Factor $=5.23 \quad$ UGC Approved [no.20489] ISSN: 2454-6089 (Online)

\begin{tabular}{|c|c|c|c|c|c|c|c|c|c|c|c|c|}
\hline 2 & 42 & 41 & 08 & 09 & 1764 & 1681 & 64 & 81 & 83 & 17 & 6889 & 289 \\
\hline 3 & 34 & 33 & 16 & 17 & 1156 & 1089 & 256 & 289 & 67 & 33 & 4489 & 1089 \\
\hline 4 & 35 & 34 & 15 & 16 & 1225 & 1156 & 225 & 256 & 69 & 31 & 4761 & 961 \\
\hline 5 & 44 & 43 & 06 & 07 & 1936 & 1849 & 36 & 49 & 87 & 13 & 7569 & 169 \\
\hline 6 & 42 & 41 & 08 & 09 & 1764 & 1681 & 64 & 81 & 83 & 17 & 6889 & 289 \\
\hline \multirow[t]{2}{*}[\mathrm{n}=6]{} & \multicolumn{2}{|c|}{$\sum P=466$} & \multicolumn{2}{|c|}{$\sum Q=134$} & $\sum \mathbf{P}^{2}=$ & 3266 & \multicolumn{2}{|c|}{$\sum Q^{2}=1666$} & \multicolumn{2}{|c|}{$\sum \mathbf{T i}^{2}=$} & 36526 & 3326 \\
\hline & \multicolumn{2}{|c|}{$\left(\sum P\right)^{2}=217156$} & \multicolumn{2}{|c|}{$\begin{array}{c}\left(\sum Q\right)^{2}= \\
17956\end{array}$} & \multicolumn{7}{|c|}{$*$ Reliability $=0.99$} & \\
\hline
\end{tabular}

\begin{tabular}{|c|c|c|c|c|c|c|c|c|c|c|c|c|}
\hline \multirow{3}{*}{ Variable } & \multicolumn{4}{|c|}{ Body Mass Index } & \multicolumn{2}{|c|}{$\mathbf{P}^{2}$} & \multicolumn{2}{|c|}{$\mathbf{Q}^{2}$} & \multicolumn{2}{|c|}{$\mathbf{T i}$} & \multicolumn{2}{|c|}{$\mathbf{T i}^{2}$} \\
\hline & \multicolumn{2}{|c|}{$\begin{array}{c}\mathbf{P} \\
{[\text { Yes] }}\end{array}$} & \multicolumn{2}{|c|}{$\begin{array}{c}\mathbf{Q} \\
{[\mathrm{No}]}\end{array}$} & \multirow[t]{2}{*}{$\mathbf{R}_{1}$} & \multirow{2}{*}{$\mathbf{R}_{\mathbf{2}}$} & \multirow{2}{*}{$\mathbf{R}_{1}$} & \multirow{2}{*}{$\mathbf{R}_{2}$} & \multicolumn{2}{|c|}{$\mathbf{R}_{\mathbf{1}} \sum \mathbf{R}_{\mathbf{2}}$} & \multicolumn{2}{|c|}{$\mathbf{R}_{1} \sum \mathbf{R}_{\mathbf{2}}$} \\
\hline & $\mathbf{R}_{1}$ & $\mathbf{R}_{2}$ & $\mathbf{R}_{1}$ & $\mathbf{R}_{2}$ & & & & & $\mathbf{P}$ & $\mathbf{Q}$ & $\mathbf{P}$ & $\mathbf{Q}$ \\
\hline 1 & 36 & 35 & 14 & 15 & 1296 & 1225 & 196 & 225 & 71 & 29 & 5041 & 841 \\
\hline 2 & 42 & 41 & 08 & 09 & 1764 & 1681 & 64 & 81 & 83 & 17 & 6889 & 289 \\
\hline 3 & 48 & 47 & 02 & 03 & 2304 & 2209 & 04 & 09 & 95 & 05 & 9025 & 25 \\
\hline 4 & 40 & 39 & 10 & 11 & 1600 & 1521 & 100 & 121 & 79 & 21 & 6241 & 441 \\
\hline 5 & 33 & 32 & 17 & 18 & 1089 & 1024 & 289 & 324 & 65 & 35 & 4225 & 1225 \\
\hline 6 & 38 & 37 & 12 & 13 & 1444 & 1369 & 144 & 169 & 75 & 25 & 5625 & 625 \\
\hline \multirow[t]{2}{*}[\mathrm{n}=6]{} & \multicolumn{2}{|c|}{$\sum P=468$} & \multicolumn{2}{|c|}{$\begin{array}{c}\sum \mathbf{Q}= \\
132\end{array}$} & \multicolumn{2}{|c|}{$\sum P^{2}=18526$} & \multicolumn{2}{|c|}{$\sum Q^{2}=1726$} & & & \multicolumn{2}{|c|}{370463446} \\
\hline & \multicolumn{2}{|c|}{$[\Sigma P]^{2}=219024$} & \multicolumn{2}{|c|}{$\begin{array}{c}{\left[\sum Q\right]^{2}=} \\
17424\end{array}$} & \multicolumn{8}{|c|}{$*$ Reliability $=0.99$} \\
\hline
\end{tabular}


Table 3. Knowledge about knowing and monitoring of vital sign of Blood Pressure [n=150]

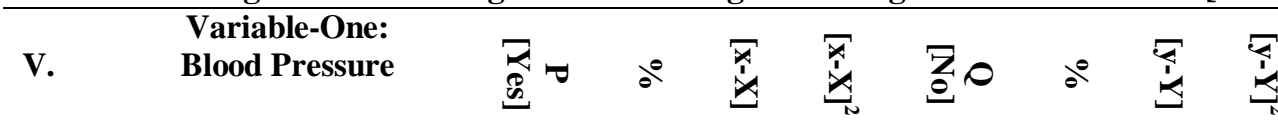

1 Do you know thatBlood Pressure [BP]as one of the vital signscan be linked to the development of low arterial pressure causing eating disorders, particularly anorexia nervosa and bulimia nervosa?

2 Do you know that Blood Pressure [BP] as one of the vital signs can be linked to the development of low arterial pressure, especially low pulse pressure, causing a sign of shock and contributes to and reflects decreased perfusion?

3 Do you know that Blood Pressure [BP] as one of the vital signs can be linked to the development of too low pressure causing hypotension, with a medical concern if it causes signs or symptoms, such as dizziness, fainting, or in extreme cases, shock?

4 Do you believe that Blood Pressure [BP] as one of the vital signs can be linked to high pressure causing hypertension, which can speed up ageing process amongst athletes if not known and monitored?

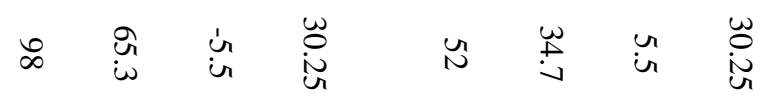

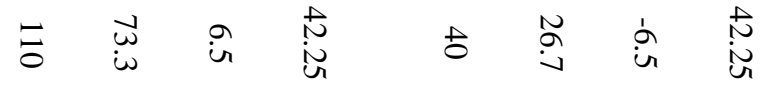

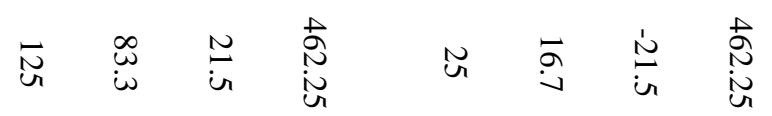



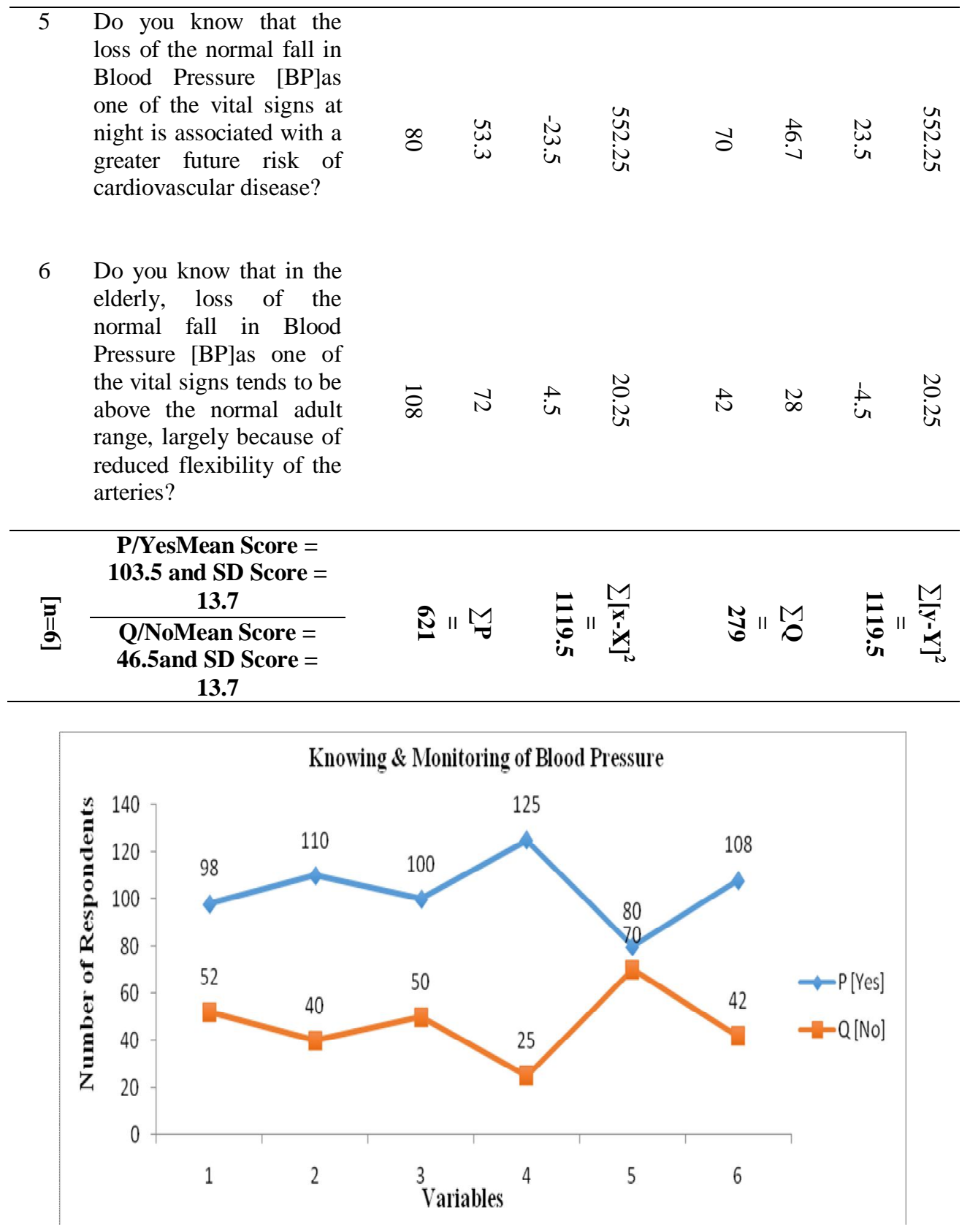

Figure 1. Knowledge about knowing and monitoring of vital sign of Blood Pressure [n=150] 
Journal of Exercise Science \& Physiotherapy, Vol. 13, No. 1, 2017

ISSN: 0973-2020 (Print) $\quad \mathrm{I}_{2}$ OR Impact Factor $=5.23 \quad$ UGC Approved [no.20489] ISSN: 2454-6089

(Online)

Table 4. Dependent t-test $(t)$ analysis about knowing and monitoring of vital sign of

Blood Pressure [n=150]

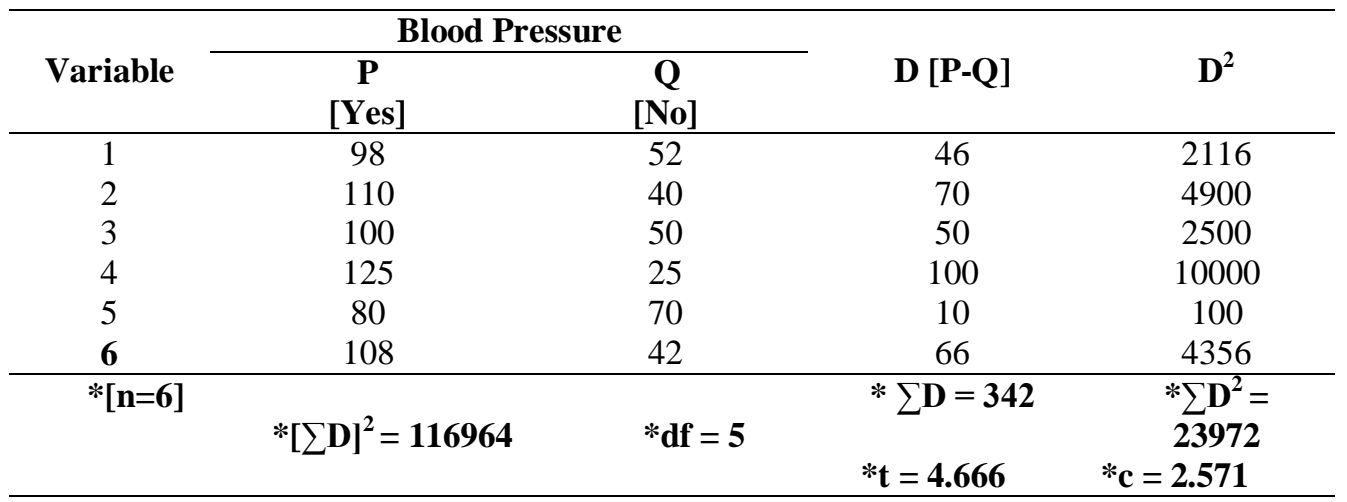

Table 5. Knowledge about knowing and monitoring vital sign of Heart Rate [n=150]

\begin{tabular}{|c|c|c|c|c|c|c|c|c|c|}
\hline V. & $\begin{array}{c}\text { Variable-Two: Heart } \\
\text { Rate }\end{array}$ & $\underset{8}{\mathbb{R}}$ & $d^{9}$ & $\underset{\ddot{x}}{\ddot{x}}$ & $\underset{N}{\stackrel{x}{x}}$ & $z_{\theta}^{Z}$ & $d^{9}$ & 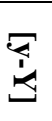 & $\underset{⿱ 乛}{\stackrel{4}{4}}$ \\
\hline 1 & $\begin{array}{l}\text { Do you know that } \\
\text { Arrhythmias } \\
\text { abnormalities of the heart } \\
\text { rate [HR] sometimes felt } \\
\text { as palpitations, which } \\
\text { produce more serious } \\
\text { symptoms } \\
\text { lightheadedness, dizziness } \\
\text { and fainting? }\end{array}$ & $\stackrel{\infty}{\sigma}$ & $\begin{array}{l}u \\
i\end{array}$ & N & $\underset{\substack{\infty \\
\perp}}{+}$ & $\stackrel{9}{\perp}$ & $\stackrel{\stackrel{N}{V}}{v}$ & N & $\underset{+\infty}{+\infty}$ \\
\hline 2 & $\begin{array}{l}\text { Has it occurred to you that } \\
\text { knowing and monitoring } \\
\text { your heart rate }[\mathrm{HR}] \text { as one } \\
\text { of the vital signs can } \\
\text { minimize the speed of } \\
\text { ageing process amongst } \\
\text { athletes? }\end{array}$ & $\vec{\infty}$ & $\underset{\sim}{\infty}$ & $\bar{\sigma}$ & $\overline{8}$ & w & $\stackrel{N}{\sim}$ & $\dot{0}$ & $\overline{8}$ \\
\hline 3 & $\begin{array}{l}\text { Are you aware that } \\
\text { athletes prone to the attack } \\
\text { of heart rate }[\mathrm{HR}] \text { as one of } \\
\text { the vital sings suffer from } \\
\text { chest pain or chest } \\
\text { discomforts? }\end{array}$ & N & $\stackrel{\infty}{\circ}$ & $\bar{N}$ & $\overrightarrow{+}$ & $\underset{\sigma}{\omega}$ & $\tilde{O}$ & $\dot{\grave{N}}$ & $\vec{f}$ \\
\hline
\end{tabular}




\section{Do you believe that athletes prone to the attack of heart rate $[\mathrm{HR}]$ as one of the vital signs can speeds up their ageing process? \\ 5 Do you know that athletes prone to the attack of heart rate $[\mathrm{HR}]$ as one of the vital signs are at risk of pain in the arm[s], back, neck, or jaw?}

6 Do you know that athletes prone to the attack of heart rate $[\mathrm{HR}]$ as one of the vital signs are also at risk ofshortness of breath, fatigue, stomach pain, nausea or lightheadedness, and sweating?

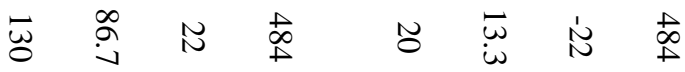

\section{P/YesMean Score $=$}

\begin{tabular}{|c|c|c|c|}
\hline $\begin{array}{c}\text { 108and SD Score }=15.7 \\
\text { Q/NoMean Score }= \\
\text { 42and SD Score }=15.7\end{array}$ & $\underset{\infty}{\infty} \| M$ & $\stackrel{\frac{M}{x}}{\frac{E}{N}}$ & 岕 " \\
\hline
\end{tabular}

Figure 2. Knowledge about knowing and monitoring vital sign of Heart Rate [n=150]

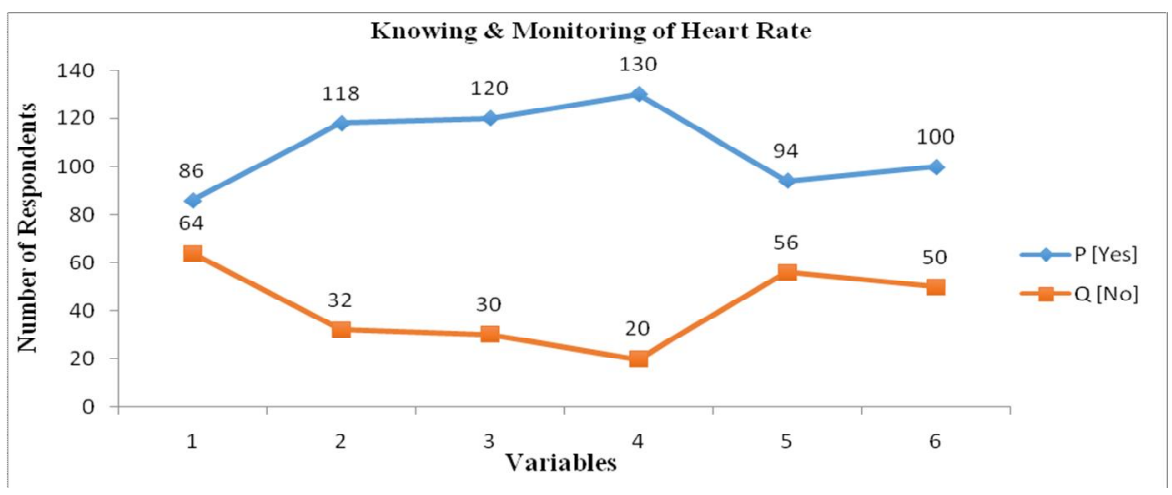


Journal of Exercise Science \& Physiotherapy, Vol. 13, No. 1, 2017

ISSN: 0973-2020 (Print) I I OR Impact Factor $=5.23$ UGC Approved [no.20489]

(Online)

Table 6. Dependent t-test $[t]$ analysis of about knowing and monitoring vital sign of Heart Rate $[\mathrm{n}=150]$

\begin{tabular}{|c|c|c|c|c|}
\hline \multirow[t]{2}{*}{ Variable } & \multicolumn{2}{|c|}{ Heart Rate } & \multicolumn{2}{|c|}{ D } \\
\hline & $\begin{array}{c}\mathbf{P} \\
{[\text { Yes] }}\end{array}$ & $\begin{array}{c}\mathbf{Q} \\
{[\mathrm{No}]}\end{array}$ & {$[\mathbf{P}-\mathbf{Q}]$} & $\mathrm{D}^{2}$ \\
\hline 1 & 86 & 64 & 22 & 484 \\
\hline 2 & 118 & 32 & 86 & 7396 \\
\hline 3 & 120 & 30 & 90 & 8100 \\
\hline 4 & 130 & 20 & 110 & 12100 \\
\hline 5 & 94 & 56 & 38 & 1444 \\
\hline 6 & 100 & 50 & 50 & 2500 \\
\hline & & & $* \sum \mathbf{D}=\mathbf{3}$ & $* \sum D^{2}=32024$ \\
\hline$*[n=6]$ & $*\left[\sum \mathbf{D}\right]^{2}$ & $* \mathbf{d f}=5$ & $* t=4.711$ & $*_{c}=2.571$ \\
\hline
\end{tabular}

Table 7. Knowledge about knowing and monitoring vital sign of Body Mass Index [n=150]

\begin{tabular}{|c|c|c|c|c|c|c|c|c|c|}
\hline V. & $\begin{array}{l}\text { Variable-Three: } \\
\text { Body Mass Index }\end{array}$ & $\underset{8}{8}$ & $a^{9}$ & $\underset{\ddot{x}}{\ddot{x}}$ & 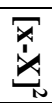 & $\underset{\theta}{z} 0$ & $0^{9}$ & 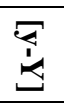 & 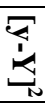 \\
\hline 1 & $\begin{array}{l}\text { Do you know that overweight and obese athletes } \\
\text { or individuals under the vital sign of body mass } \\
\text { index [BMI] are at an increased risk of } \\
\text { hypertension? }\end{array}$ & ö & $\overrightarrow{0}$ & $\bar{\infty}^{\prime}$ & $\underset{\perp}{\infty}$ & 它 & $\ddot{\sigma}$ & $\stackrel{\dot{\infty}}{\infty}$ & $\underset{\perp}{+}$ \\
\hline 2 & $\begin{array}{l}\text { Do you believe that overweight and obesity } \\
\text { under the vital sign of body mass index } \\
\text { [BMI]can speed } \\
\text { processamongstathletes? }\end{array}$ & $\breve{w}_{u}$ & 8 & $\stackrel{\omega}{\infty}$ & 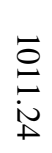 & ur & 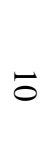 & $\stackrel{\dot{\omega}}{\infty}$ & 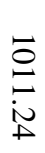 \\
\hline 3 & $\begin{array}{l}\text { Are you aware that athletes prone to overweight } \\
\text { and obesity under the vital sign of body mass } \\
\text { index [BMI]suffer fromdiseases like coronary } \\
\text { heart disease? }\end{array}$ & י & $\stackrel{a}{\omega}$ & $\stackrel{\dot{1}}{\dot{n}}$ & $\begin{array}{l}\vec{N} \\
\stackrel{\leftrightarrow}{+} \\
\dot{D}\end{array}$ & $\ddot{\infty}$ & $\stackrel{w}{\infty}$ & $\overline{\text { i }}$ & 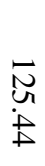 \\
\hline 4 & $\begin{array}{l}\text { Knowing and monitoring of vital signs like body } \\
\text { mass index [BMI]; can this minimize the speed } \\
\text { of ageing process amongst athletes? }\end{array}$ & $\bar{\infty}$ & $\stackrel{\infty}{\sim}$ & $\underset{\infty}{+\infty}$ & $\begin{array}{l}\stackrel{N}{O} \\
\dot{\circ} \\
+\end{array}$ & $\underset{N}{w}$ & $\stackrel{N}{\omega}$ & $\underset{\infty}{+}$ & 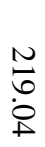 \\
\hline 5 & $\begin{array}{l}\text { Do you know thattype II diabetes and stroke are } \\
\text { also increased risk factors under the vital sign of } \\
\text { body mass index [BMI]for overweight and } \\
\text { obese athletes? }\end{array}$ & $\infty$ & 崩 & $\stackrel{\vec{H}}{\stackrel{i}{N}}$ & $\begin{array}{l}\stackrel{N}{\circ} \\
\stackrel{9}{+}\end{array}$ & 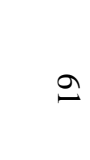 & $\stackrel{f}{\bullet}$ & $\vec{i}$ & 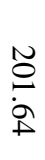 \\
\hline 6 & $\begin{array}{l}\text { Do you also know that gallbladder disease and } \\
\text { osteoarthritis are increased risk factors under the } \\
\text { vital sign of body mass index [BMI] for } \\
\text { overweight and obese athletes? }\end{array}$ & $\stackrel{\infty}{\circ}$ & $\begin{array}{l}u \\
w \\
i\end{array}$ & 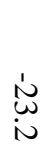 & $\begin{array}{l}\underset{\omega}{W} \\
\infty \\
\dot{\perp} \\
+\infty\end{array}$ & d & 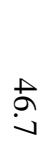 & $\bigcup_{i}^{N}$ & S \\
\hline & P/YesMean Score $=\mathbf{1 0 3 . 2}$ and SD Score $=18.7$ & \multirow{2}{*}{\multicolumn{4}{|c|}{ 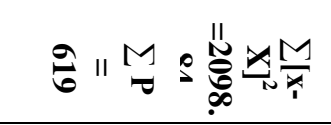 }} & \multirow{2}{*}{\multicolumn{4}{|c|}{ N }} \\
\hline$\hat{\theta}$ & Q/NoMean Score $=46.8$ and SD Score $=18.7$ & & & & & & & & \\
\hline
\end{tabular}




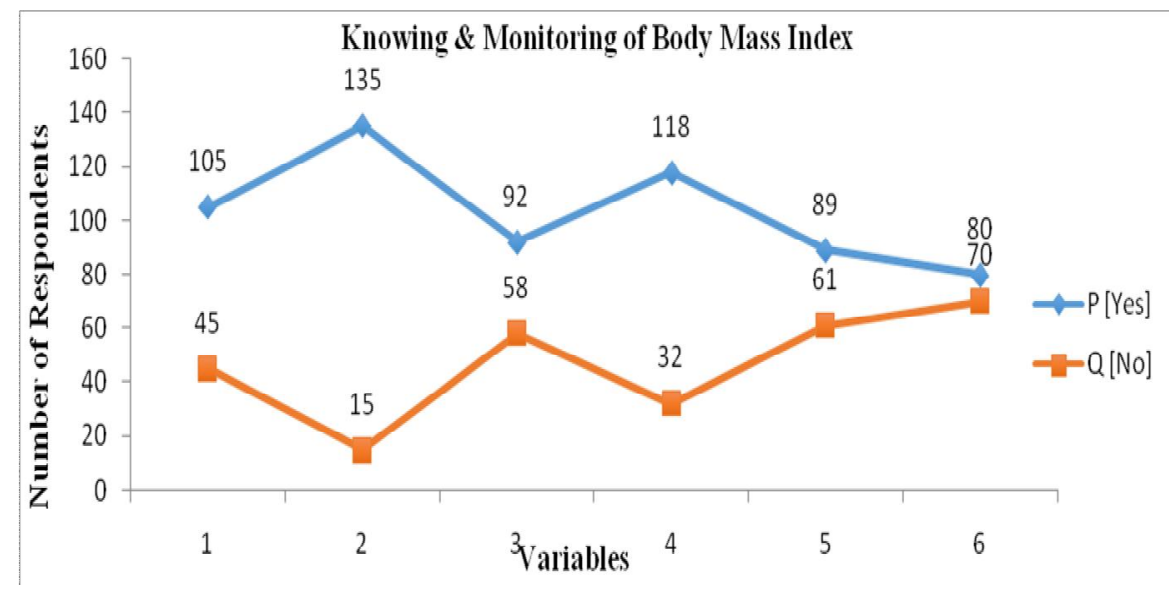

Figure 3. Knowledge about knowing and monitoring vital sign of Body Mass Index [n=150]

Table 8. Dependent t-test ( $t$ ) analysis of knowing and monitoring vital sign of Body Mass Index $[\mathbf{n}=\mathbf{1 5 0}]$

\begin{tabular}{|c|c|c|c|c|}
\hline \multirow[t]{2}{*}{ Variable } & \multicolumn{2}{|c|}{ Body Mass Index } & \multirow[b]{2}{*}{$\begin{array}{c}\text { D } \\
{[\mathbf{P}-\mathbf{Q}]}\end{array}$} & \multirow[b]{2}{*}{$D^{2}$} \\
\hline & $\begin{array}{c}\mathbf{P} \\
{[\text { Yes] }}\end{array}$ & $\begin{array}{c}\mathbf{Q} \\
{[\mathrm{No}]}\end{array}$ & & \\
\hline 1 & 105 & 45 & 60 & 3600 \\
\hline 2 & 135 & 15 & 120 & 14400 \\
\hline 3 & 92 & 58 & 34 & 1156 \\
\hline 4 & 118 & 32 & 86 & 7396 \\
\hline 5 & 89 & 61 & 28 & 784 \\
\hline 6 & 80 & 70 & 10 & 100 \\
\hline$*[n=6]$ & $*\left[\sum \mathbf{D}\right]^{2}$ & $* d f=5$ & $\begin{array}{l}* \sum D=338 \\
* t=3.368\end{array}$ & $\begin{array}{c}* \sum D^{2}= \\
27436 \\
* c=2.571\end{array}$ \\
\hline
\end{tabular}

\section{Discussion}

The survey only examined the knowledge of University Athlete about knowing and monitoring of vital signs of blood pressure [BP], heart rate [HR] and body mass index [BMI] as preventive strategy in reducing early and unsuccessful ageing cased at Njala Campus. With an increasing effort by researchers in a bid of seeking possible and vital solutions in reducing early and unsuccessful ageing in persons, Schriger, D. L. (2007) reported that, blood pressure, heart rate, body temperature and body mass index among others are measures of vital signs, that varies according to a person's age, weight, gender and overall health taken to help assess the general physical health of a person, give clues to possible diseases, and show progress toward recovery. Statistical instrument used: Percentage [\%], Dependent t-test [t], Standard Deviation, Mean, Tabulated Frequency Distribution and Chart were comparatively used in the analysis and testing of the findings at level of significance $\mathrm{p}<0.05$. 


\section{Journal of Exercise Science \& Physiotherapy, Vol. 13, No. 1, 2017 \\ ISSN: 0973-2020 (Print) I2OR Impact Factor $=5.23$ UGC Approved [no.20489] ISSN: 2454-6089 \\ (Online)}

In discussing the objective of the survey aimed at University Athletes knowledge bout knowing and monitoring of vital signs of Blood Pressure (BP), Heart Rate (HR) and Body Mass Index (BMI) as primary preventive strategy in reducing early and unsuccessful ageing, cased at Njala Campus, the findings were of great significant with positively skewed values for all the variables measuredas tabled in 4, 6 and 8, [t-values of 4.666, 4.711 and 3.368] when tested at $p<0.05$. Knowing and monitoring of vital sign of blood pressure at home as positively skewed variable in one of the findings, will help maintained blood pressure state of equilibrium among persons (University Athletes) as put forward by Chobanian, A. V. et al (2003) which state that, knowing and monitoring of blood pressure will improve hypertension management and monitor the effects of lifestyle changes and medication related to blood pressure. However, ambulatory monitoring according to Shimbo, D. et al. (2007) is recommended for most patients at home before the start of antihypertensive drugs. In the same vein, Gottdiener, J. S. et al (2002)reported that, shock as a main factor of blood pressure is a complex condition, which leads to critical decreased perfusion leading to the loss of blood volume and pooling of blood within the veins reducing adequate return to the heart and/or low effective heart pumping causing lightheadedness, dizziness, and weakness or fainting. Also, skewed positively in the above finding is the knowing and monitoring of heart rate, which according to Karvonen, J. et al. (1988), is not a stable value and it increases or decreases in response to the body's need in a way to maintain equilibrium (basal metabolic rate) between requirement and delivery of oxygen and nutrients. The normal SAN firing rate is affected by autonomic nervous system activity: sympathetic stimulation increases and parasympathetic stimulation decreases the firing rate. Regarding body mass index, the result was also positively skewed as indicated in the responses of the participants though according to Schneider, H. J. et al. (2010), in their 2010 study that followed 11,000 subjects for up to eight years, BMI is not a good measure for the risk of heart attack, stroke or death due to its simplicity. Nonetheless, looking at the responses tabled in 3,5 and 7 and figured in 1, 2 and 3 above, the findings indicate a highly positive skewed view of University Athletes knowledge bout knowing and monitoring of vital signs of Blood Pressure (BP), Heart Rate (HR) and Body Mass Index (BMI) as primary preventive strategy in reducing early and unsuccessful ageing cased at Njala Campus, as proved in their respective scores of percentages, mean and standard deviation.

\section{Conclusion}

In the conclusion summary, the survey only looked at the assessment of University Athletes knowledge about the knowing and monitoring of vital signs of blood pressure [BP], heart rate [HR] and body mass index [BMI], as preventive strategy in reducing early and unsuccessful ageing and recommend as necessary. As embedded in the findings above with special reference to the t-scores, percentage scores, and mean scores, the entire results were skewed positively leaving undeniable justification about University Athletes knowledge in knowing and monitoring of vital signs of Blood Pressure (BP), Heart Rate (HR) and Body Mass Index (BMI) as primary preventive strategy in reducing early and unsuccessful ageing cased at Njala Campus. This according to Muffuli, et al. (2003) is referred to as primary prevention knowledge in reducing progressive early and unsuccessful ageing among athletes favouring active and healthy ageing.

The survey recommends thus: That further research be carried out about other areas of vital signs perhaps with more variables and greater number of participants to help deepened the knowledge of athletes in that direction and for a better generalization of findings. Also, that University Athletes should be more proactive in their frequent home monitoring and evaluation in a way to establish a state of equilibrium regarding vital signs which according to report from, National Early Warning Score Development and Implementation Group (2012), are measurements taken to help assess the general physical health of a person, give clues to possible diseases, and show progress toward 


\section{Journal of Exercise Science \& Physiotherapy, Vol. 13, No. 1, 2017 \\ ISSN: 0973-2020 (Print) I2OR Impact Factor =5.23 UGC Approved [no.20489] ISSN: 2454-6089 \\ (Online)}

recovery. And that training workshops be made readily available to University Athletes, cased at Njala Campus in the area of knowing and monitoring of vital signs of Blood Pressure (BP), Heart Rate (HR) and Body Mass Index (BMI) as primary preventive strategy in reducing early and unsuccessful ageing.

Acknowledgement

The authors express sincere thanks and appreciation to all staff and students of Njala University, Njala Campus, whose immense co-operation rendered this study to fruition.

References

Acute Care Toolkit. (2013). The medical patient at risk: recognition and care of the seriously ill or deteriorating medical patient" (PDF): Royal College of Physicians of London.

Ademola, A. (2011). Health risk behaviours of elite athletes in Oyo State, Journal of Nigeria Association for Physical, Health Education, Recreation, Sport and Dance, 2(1): 39-44

Alfredson, H. and Lorentzon, R. (2012). Chronic Achilles Tendinosis: Recommendations for Treatment and Prevention. Sports Medicine, 29, 135-146.

American Heart Association. (2011)."Understanding blood pressure readings".

American Heart Association and American College of Sports Medicine (2007). Joint Position Statement: Exercise and acute cardiovascular events: placing the risks into perspective. Med. Sci. Sports Exerc: 39:886-897.

American Psychiatry Association. (2013).Diagnostic and Statistical Manual of Mental Disorders ( $^{\text {th }}$ Ed.) Arlington: American Psychiatric Publishing. pp. 329-354. ISBN 0890425558.

American Psychiatric Association. (2013)..Diagnostic and Statistical Manual of Mental Disorders ( ${ }^{\text {th }}$ Ed.). Arlington, V. A: American Psychiatric Publishing. pp. 345-349. ISBN 978-089042-555-8.

American Psychiatric Publishing. (2013)..Diagnostic and statistical manual of mental disorders: $D S M-5$ ( $5^{\text {th }}$ Ed.). Washington: pp. 338-345. ISBN 9780890425558.

Anderson-Fye, E. P. and Becker, A. E. (2004). "Sociocultural Aspects of Eating Disorders" pp. 56589 in Handbook of Eating Disorders and Obesity, J. Kevin (ed.). Thompson. Hoboken, NJ: John Wiley \& Sons.

Anderson, J. M. (1991)."Rehabilitating elderly cardiac patients". West. J. Med.154 (5): 573-8.

Andrew, W. (2003).Masters of Theory: Cambridge and the Rise of Mathematical Physics, page 213, University of Chicago Press ISBN 0-226-87374-9

Arcelus, J., Witcomb, G. L. and Mitchell, A. (2014). "Prevalence of eating disorders amongst dancers: a systemic review and meta-analysis." European eating disorders review: the journal of the Eating Disorders Association:22 (2): 92-101.

Artero, E.,España-Romero, V., Ortega, F., Jiménez-Pavón, D., Ruiz, J., Vicente-Rodríguez G., Bueno, M., Marcos, A., Gómez-Martínez, S., Urzanqui, A., González-Gross, M., Moreno, L., Gutiérrez, A. and Castillo, M. (2010).Health-related fitness in adolescents: underweight, and not only overweight, as an influencing factor. The AVENA study: Scand J Med Sci Spor: 20 (3): 418-427.

Attia, E. (2010). "Anorexia Nervosa: Current Status and Future Directions". Annual Review of Medicine:61 (1): 425-35.

Baggaley, R. F., White R. G. and Boily, M. C. (2008). "Systematic review of orogenital HIV-1 transmission probabilities." International Journal of Epidemiology:37 (6): 1255-65.

Banks, A.J., Shepard, G.J. and Ryan, W.G. (2003). prevalence of osteoarthritis of the hip compared with age matched controls in ex-professional footballers. British journal of sports medicine 37(1): 80-81.

Barbaro, G. and Barbarini, G. (2011) "Human immunodeficiency virus \& cardiovascular risk". The Indian journal of medical research:134 (6): 898-903.

Barker, P. (2003)Psychiatric and Mental Health Nursing: The Craft of Caring. Great Britain: Arnold. ISBN 0340810262. 


\section{Journal of Exercise Science \& Physiotherapy, Vol. 13, No. 1, 2017 \\ ISSN: 0973-2020 (Print) $\quad \mathrm{I}_{2}$ OR Impact Factor $=5.23 \quad$ UGC Approved [no.20489] ISSN: 2454-6089 \\ (Online)}

Baum, A. and Auckland, N. Z (2006). "Eating Disorders in the Male Athlete" (PDF). Sports medicine:36 (1): 1-6.

Bebeley, S. J. (2016).Athletes' Knowledge about Preventing Sports Injuries like: Achilles Tendinitis Runner's Knee/Patellofemoral Pain Syndrome and Shin Splints, as Prime Prevention Strategies in Slowing Ageing Process. Journal of Exercise Science and Physiotherapy: 12 (1): 22-28.

Berrigan, D., Dodd, K., Troiano, R., Krebs-Smith, S.M. and Barbash, R.B. (2003): Patterns of health behaviour in US adults. Prev. Med. 36, 615-623.

Blagosklonny, M. V. (2009). Validation of anti-ageing drugs by treated diseases. Ageing 1 (3): 281 8 ,

Bonci, C. M., Bonci, L. J., Granger, L. R., Johnson, C. L., Malina, R. M., Milne, L. W., Ryan, R. R. and Vanderbunt, E. M. (2008). "National athletic trainers' association position statement: Preventing, detecting, and managing disordered eating in athletes". Journal of Athletic Training 43 (1): 80-108.

Bowen, R. L. and Atwood, C. S. (2004). "Living and Dying for Sex". Gerontology:50 (5): $265-90$.

Bradley, C. B., McMurray, R. G., Harrell, J. S., \& Deng, S. (2000): Changes in common activities of $3^{\text {rd }}$ through $10^{\text {th }}$ graders: the CHIC study. Medicine and Science in Sports and Exercise: 32 , 2071-2078

Brewerton, T. (2014). "Binge Eating: Recognition, Diagnosis, and Treatment". Medscape Health Journal: Retrieved 2014.

Bulik, C. M., Marcus, M. D., Zerwas, S., Levine, M. D. and La Via, M. (2012). "The changing "weightscape" of bulimia nervosa." The American Journal of Psychiatry:169 (10): 10316.

"Bulimia nervosa fact sheet" (2012).Office on Women's Health.

Cantoni, G. (1998): The Road to an Aging Policy for the 21st Century: ISBN 978-0-7881-4635-0

Centres for Disease Control and Prevention (CDC), (2001). Increasing physical activity: a report on recommendations on the Task Force on Community Preventive Services: MMWR 50:1-14.

Centers for Disease Control and Prevention (CDC). (2003). "HIV and Its Transmission" Archived from the original 2005 Retrieved (2006).

Centers for Disease Control and Prevention (CDC). (2006). "Expedited partner therapy in the management of sexually transmitted diseases".

Chobanian, A. V., Bakris, G. L., Black, H. R., Cushman, W. C., Green, L. A., Izzo, J. L., Jones, D. W., Materson, B. J., Oparil, S., Wright, J. T. and Roccella, E. J. (2003)."Seventh report of the Joint National Committee on Prevention, Detection, Evaluation, and Treatment of High Blood Pressure". Hypertension:42 (6): 1206-52.

Colfer, G. R. (2004). "Skill-related physical fitness essential for sports success" tradoc.army.mil. Archived from the original 2011

Conry, M. C., Morgan, K. and Curry, P. et al. (2011). The clustering of health behaviours in Ireland and their relationship with mental health, self-rated health and quality of life. BMC Public Health: 11, 692.

Coups, E., Gaba, A. and Orleans, T. (2004). Physician screening for multiple health risk factors. Am. J. Prev. Med. 27, 34-41.

Deakin, C. D. and Low, J. L. (2000)."Accuracy of the advanced trauma life support guidelines for predicting systolic blood pressure using carotid, femoral, and radial pulses: observational study". BMJ:321(7262): 673-4.

de Groot, Gudrun Cathrine Lindgren; Fagerström, Lisbeth (2010). "Older adults' motivating factors and barriers to exercise to prevent falls". Scandinavian Journal of Occupational Therapy:18 (2): 153-160.

Department of Health, Education, and Welfare, (1972). A Common Thread of Service: A Historical Guide to HEW. DHEW Publication No (OS): 73-45 http://aspe.hhs.gov/info/hewhistory.htm (2014). 


\section{Journal of Exercise Science \& Physiotherapy, Vol. 13, No. 1, 2017 \\ ISSN: 0973-2020 (Print) $\quad$ I $_{2}$ OR Impact Factor $=5.23$ UGC Approved [no.20489] ISSN: 2454-6089 \\ (Online)}

D'Hont, E., Deforche, B., Bourdeaudhuij, I. and Lenoir, M. (2009). Relationship between motor skill and Body Mass Index in 5-to 10-year-old children: Adapt Phys Act Q. 26:21-37.

Dillin, A., Gottschling, D. E., Nyström, T. and Gottschling, N. (2014). "The good and the bad of being connected: the integrons of aging". CurrOpin Cell Biol:26: 107-12.

Dixit, S., DiFiori, J. P., Burton, M. and Mines, B. (2007). "Management of patellofemoral pain syndrome" American family physician: 75 (2): 194-202.

Donatelle, R. (2009). Promoting Healthy Behavior Change. Health: The basics: (pp. 4). 8th edition. San Francisco, CA: Pearson Education, Inc.

Dorland, A. (2003). Health, wellness and disability. Oregon Health and Science University Press

Douglas, H. (2001). "Online Etymology Dictionary: bulimia". Online Etymology Dictionary.

Dumith, S., Ramires, V., Souza, M., Moraes, D., Petry, F., Oliveira, E., Ramires, S. andHallal, P. (2010).Overweight/Obesity and physical fitness among children and adolescents. $J$ Phys Act Health: 7:641-648

Eating disorders and culture" (2004).Harvard Mental Health Letter20 (9): 7.

Evans, M., Weinberg, R., and Jackson, A. (1992). Psychological factors related to drug use in college athletes. The Sport Psychologist: 6 24-41.

Eknoyan and Garabed (2007). "Adolphe Quetelet (1796-1874)-the average man and indices of obesity". Nephrology Dialysis Transplantation:23 (1): 47-51

Espi, F. F. (2013). "Anorexia mirabilis: the practice of fasting by Saint Catherine of Siena in the late middle Ages". American Journal of Psychiatry: 170 (4): 370-1.

Fairburn, and Christopher, (2013).Overcoming binge eating: the proven program to learn why you binge and how you can stop (Second ed.). New York: Guilford Publications: ISBN 1572305614.

Fenwick, S. A., Hazleman, B. L., \& Riley, G. P. (2002). The vasculature and its role in the damaged and healing tendon. Arthritis Research: 4(4), 252-260.

Fine, L., Philogene, G., Gramling, R. and Coups, E. (2004). Prevalence of multiple chronic disease risk factors: 2001 National Health Interview Survey. Am. J. Prev. Med. 27, 18-24.

Franko, D. L., Becker, A. E., Thomas, J. J., Herzog, D. B., Becker; Thomas; Herzog (2007). "Crossethnic differences in eating disorder symptoms and related distress" The International Journal of Eating Disorders:40 (2): 156-64.

French, S., Rosenberg, M. and Knuiman, M. (2008). The clustering of health risk behaviours in a Western Australian adult population. Health Promot. J. Austr. 19, 203-209.

Fries, J. F. (2002). "Reducing Disability in Older Age". JAMA:288 (24): 3164-6.

Gao, L., Zhang, L. and Jin, Q. (2009). "Meta-analysis: prevalence of HIV infection and syphilis among MSM in China." Sexually transmitted infections:85 (5): 354-8.

Gerdy, R. John. (2000). "Sports in School the Future of an Institution". Teachers College Press.

Giannini, A. J. (1993). "A history of bulimia". In The Eating Disorders: (pp. 18-21) Springer New York.

Gottdiener, J. S., Panza, J. A., St John Sutton, M., Bannon, P., Kushner, H. and Weissman, N. J. (2002)."Testing the test: The reliability of echocardiography in the sequential assessment of valvular regurgitation". American Heart Journal: 144 (1): 115-21 Retrieved (2010).

Grad, F. P. (2002). "The Preamble of the Constitution of the World Health Organization". Bulletin of the World Health Organization:80 (12): 982.

Gull, Sir William Withey (1894). T. D. Acland, ed. Medical Papers. p. 309.

Gull, W. W. (1997). "Anorexia nervosa (apepsiahysterica, anorexia hysterica). 1868." Obesity research:5 (5): 498-502.

Hanaola, T. (2005). Survey in smoking behaviour, knowledge and attitudes towards smoking and health of dental students. Journal of dental health: 55(2): 100-108.

Hay, P. (2013). "A systematic review of evidence for psychological treatments in eating disorders: 2005-2012." The International Journal of Eating Disorders:46 (5): 462-9.

Hay, P. J. and Claudino, A. M. (2010). "Bulimia nervosa". Clinical Evidence2010: 1009.

Huber, M., Knottnerus, J. A., Green, L., van der Horst, H., Jadad, A. R., Kromhout, D. and Smid, H. 


\section{Journal of Exercise Science \& Physiotherapy, Vol. 13, No. 1, 2017 \\ ISSN: 0973-2020 (Print) $\quad$ I $_{2}$ OR Impact Factor $=5.23$ UGC Approved [no.20489] ISSN: 2454-6089 \\ (Online)}

(2011). Definition of Health: BMJ: 343 (d4163)

Iacovino, J. M., Gredysa, D. M., Altman, M. and Wilfley, D. E. (2012). "Psychological treatments for binge eating disorder" Curr Psychiatry Rep:14 (4): 432-46.

Jeremy Singer-Vine (2009)."Beyond BMI: Why doctors won't stop using an outdated measure for obesity" Retrieved (2013).

Joint Committee on Terminology (2001). "Report of the 2000 Joint Committee on Health Education and Promotion Terminology". American Journal of Health Education:32 (2): 89-103.

Kallings, L. O. (2008): "The first postmodern pandemic: 25 years of HIV/AIDS". Journal of Internal Medicine:263 (3): 218-43.

Karp, G., Schlaeffer, F., Jotkowitz, A. and Riesenberg, K. (2009). "Syphilis and HIV co-infection". European journal of internal medicine:20 (1): 9-13.

Karvonen, J. and Vuorimaa, T. (1988). "Heart rate and exercise intensity during sports activitiespractical application". Sports Medicine:5 (5): 303-11.

Katz, S. and Calasanti, T. (2015). "Critical perspectives on successful aging: Does it appeal more than it illuminates?" The Gerontologist: 55(1): 26-33.

Keys, A., Fidanza, F., Karvonen, M. J., Kimura, N. and Taylor, H. L. (1972). "Indices of relative weight and obesity". Journal of Chronic Diseases:25 (6-7): 329-43.

Kirkwood, T. and Austad, S. (2000). Why do we age? Journal of nature 408: 233-238,

Larke, N. (2010). "Male circumcision, HIV and sexually transmitted infections: a review" British journal of nursing (Mark Allen Publishing): 19 (10): 629-34.

Lennox, A., Pienaar, A. and Wilders, C. (2008).Physical fitness and the physical activity status of 15-year-old adolescents in a semi-urban community: $S$ Afr J Res Sport Phys Educ Recreation: 30(1): 59-73.

Long, J. (2004). Sports and the ageing population (online): www.sportdevelopment,org.uk/hotmail

Lupien, S. J. and Wan, N. (2004)."Successful Ageing: From Cell to Self". Philosophical Transactions of the Royal Society B: Biological Sciences:359 (1449): 1413-26.

Maffulli, N., Sharma, P., \& Luscombe, K. L. (2004). Achilles tendinopathy: aetiology and management. Journal of the Royal Society of Medicine, 97(10), 472-476.

Maffuli, N., Chan, K., Macdonak, R., Malina, R.M. and Parker, A.W. (2003).Sports medicine for specific age group and abilities. Elservier health services Churchill Livingstone.

Malcolm, K., Dr. (2015)."Why being 'overweight' means you live longer: The way scientists twist the facts". http://www.independent.co.uk

Marzola, E., Nasser, J. A., Hashim, S. A., Shih, P. A. and Kaye, W. H. (2013). "Nutritional rehabilitation in anorexia nervosa: review of the literature and implications for treatment". BMC Psychiatry13 (1): 290.

Mayo Clinic staff (2009)."Low blood pressure (hypotension) - Causes"..MayoClinic.com: Mayo Foundation for Medical Education and Research; Retrieved (2010).

Mcaloney, K., Graham, H., Law, C. and Platt, L. (2013). A scoping review of statistical approaches to the analysis of multiple health-related behaviours. Prev. Med. 56, 365-371.

McBride, H. (2012). "Study Reveals Stunning Prevalence of Bulimia among African-American Girls". Archived from the original (2012).

McKenzie, J., Neiger, B. and Thackeray, R. (2009). Health Education can also be seen as preventive medicine (Marcus 2012). Health Education and Health Promotion. Planning, Implementing, \& Evaluating Health Promotion Programs: (pp. 3-4). 5th edition. San Francisco, CA: Pearson Education, Inc.

Miller, K. K. (2013). "Endocrine effects of anorexia nervosa". Endocrinol. Metab. Clinic North America:42 (3): 515-28.

Ming, Z., Criswell, H. E., Yu, G. and Breese, G. R. (2006). Competing presynaptic and postsynaptic effects of ethanol on cerebellar purkinje neurons: Alcohol, Clinical and Experimental Research: 30 (8), 1400-1407.

Mitchison, D. (2013). "Sex differences in health-related quality of life impairment associated with eating disorder features: A general population study". International Journal of Eating 


\section{Journal of Exercise Science \& Physiotherapy, Vol. 13, No. 1, 2017 \\ ISSN: 0973-2020 (Print) I2OR Impact Factor $=5.23 \quad$ UGC Approved [no.20489] ISSN: 2454-6089 \\ (Online)}

Disotders:46: 375-380.

Moen, M., Tol, J., Weir, A., Steunebrink, M. \& De Winter, T. (2009). Medial tibial stress syndrome: a critical review. American Journal of Sports Medicne; 39.7: 524-544

Mørkedal, B., Romundstad, P. R. and Vatten, L. J. (2011)."Informativeness of indices of blood pressure, obesity and serum lipids in relation to ischaemic heart disease mortality: the HUNT-II study". European Journal of Epidemiology:26 (6): 457-461.

National Early Warning Score Development and Implementation Group (2012).National Early Warning Score (NEWS): standardizing the assessment of acute-illness severity in the NHS. London: Royal College of PhysiciansISBN 978-1-86016-471-2.

National Heart Lung and Blood Institute, (2008)."Diseases and conditions index - hypotension"

National Heart, Lung and Blood Institute, (2014)."Assessing Your Weight and Health Risk"

National Institute for Health and Clinical Excellence (2007).Clinical guideline 50: Acutely ill patients in hospital. London.

Nattiv, A., \& Puffer, J.C. (1991). Lifestyles and health risks of collegiate athletes. TheJournal of Family Practice: 33 (6), 585-590.

Nauert, PhD, R. (2015). Black Girls at Risk for Bulimia. Psych Central. Retrieved from http://psychcentral.com/news/2009/03/19/black-girls-at-risk-for-bulimia/4835.html

Neilson, E. A. (1988). Health Values: Achieving high-level wellness-Origin, philosophy, purpose. Health Values: 12(3): 3-5.

Nied, R. J. and Franklin, B (2002). "Promoting and prescribing exercise for the elderly". American family physician:65 (3): 419-26.

Okuneye, R. O., Idowu, B. B. and Abiola, M. J. (2011). Knowledge of primary prevention strategies in slowing the ageing process among Lagos State University Athletes, Journal of Nigeria Association for Physical, Health Education, Recreation, Sport and Dance: 2(1): 29-38.

Ortega, F. B., Ruiz, J. R., Mesa, J. L., Gutierrez, A. and Sjostrom, M. (2007). Cardiovascular fitness in adolescents: the influence of sexual maturation status - the AVENA and EYHS studies. Am J Hum Biol: 19, 801-808.

Overman, S. J., and Terry, T. (1991). Alcohol use and attitudes: a comparison of college athletes and nonathletes. Journal of Drug Education: 21 (2), 107-117.

Pearce, J. M. (2004). "Richard Morton: Origins of Anorexia nervosa". European Neurology:52 (4): 191-192.

Peel, N. M., McClure, R. J. and Bartlett, H. P. (2005). "Behavioral determinants of healthy aging1". American Journal of Preventive Medicine:28 (3): 298-304.

Phelan, E. A. and Larson, E. B. (2002). "'Successful Aging'-Where Next?". Journal of the American Geriatrics Society:50 (7): 1306-8.

Poortinga, W. (2007). The prevalence and clustering of fourmajor lifestyle risk factors in an English adult population. Prev. Med. 44, 124-128.

Pronk, N., Anderson, L. and Crain, A. (2004). Meeting recommendations for multiple healthy lifestyle factors. Am. J. Prev. Med. 27, 25-33.

Putra, B. E. and Sutarina, N. (2014). "Combination of hip-quadriceps strengthening exercise: Is it more potential than single conventional strengthening exercise to reduce pain in patellofemoral pain syndrome patients?" Saudi Journal of Sports Medicine: 14 (1): 9-13.

Rikani, A. A., Choudhry, Z., Choudhry, A. M., Ikram, H., Asghar, M. W., Kajal, D., Waheed, A. and Mobassarah, N. J. (2013). "A critique of the literature on etiology of eating disorders.". Annals of Neurosciences:20 (4): 157-61.

Rixe, J. A., Glick, J. E., Brady, J. and Olympia, R. P. (2013). "A review of the management of patellofemoral pain syndrome." The Physician and sports medicine: 41 (3): 19-28.

Rowe, J. W. and Kahn, R. L. (1997). "Successful Aging". The Gerontologist:37 (4): 433-40.

Russell, G. (1997).The history of bulimia nervosa. D. Garner \& P. Garfinkel (Eds.), Handbook of Treatment for Eating Disorders ( $2^{\text {nd }}$ Ed., pp. 11-24). New York, NY: The Guilford Press.

Saguy, A. and Gruys, K. (2014). "Morality and Health: News Media Constructions of Overweight and Eating Disorders" (PDF). UCLA. Retrieved 2014. 


\section{Journal of Exercise Science \& Physiotherapy, Vol. 13, No. 1, 2017 \\ ISSN: 0973-2020 (Print) I2OR Impact Factor $=5.23$ UGC Approved [no.20489] ISSN: 2454-6089 \\ (Online)}

Salcedo, A., Palacios, X. and Fernanda, A. (2011). Alcohol consumption in young college students. AvancesenPsicologíaLatinoamericana 29, 77-97.

Sari Fine Shepphird (2009).100 Questions \& Answers about Anorexia Nervosa: Jones \& Bartlett Learning. p. xvi. ISBN 9781449630799.

Sepkowitz, K. A. (2001). "AIDS-the first 20 years". N. Engl. J. Med:344 (23): 1764-72.

Schmidt-Nielsen and Knut (1997).Animal physiology: adaptation and environment ( ${ }^{\text {th }}$ Ed.). Cambridge: Cambridge Univ. Press. Pp. 104:ISBN 978-0-521-57098-5.

Schneider, H. J., Friedrich, N., Klotsche, J., Pieper, L., Nauck, M. and John, U., et al. (2010). "The Predictive Value of Different Measures of Obesity for Incident Cardiovascular Events and Mortality" Journal of Clinical Endocrinology \& Metabolism:95 (4): 1777-85.

Schriger, D. L. (2007). Approach to the patient with abnormal vital signs. Goldman, L. and Ausiello, D. Cecil Textbook of Medicine. 23rd ed. Philadelphia, Pa: Saunders Elsevier: chap 7.

Shimbo, D., Pickering, T. G., Spruill, T. M., Abraham, D., Schwartz, J. E. and Gerin, W. (2007)."The Relative Utility of Home, Ambulatory, and Office Blood Pressures in the Prediction of End-Organ Damage". Am J Hypertens:20 (5): 476-82.

Smink, F. R., van Hoeken, D. and Hoek, H. W. (2012). "Epidemiology of eating disorders: incidence, prevalence and mortality rates." Current Psychiatry Reports: 14 (4): 406-14.

Strumia, R. (2009). "Skin signs in anorexia nervosa". Dermatoendocrinol:1 (5): 268-70.

Surgenor, L. J. and Maguire, S. (2013). "Assessment of anorexia nervosa: an overview of universal issues and contextual challenges". Journal of Eating Disorders:1 (1): 29.

Taanila, H., Hemminki, A., Suni, J., Pihlajamäki, H. and Parkkari, J. (2011). Low physical fitness is a strong predictor of health problems among young men: a follow-up study of 1411 male conscripts. BMC Public Health; 11: 590.

"Tachycardia| Fast Heart Rate" (2013).Tachycardia: American Heart Association.

"Target Heart Rates - AHA" (2014). Target Heart Rates: American Heart Association.

Tölgyes, T. and Nemessury, J. (2004). "Epidemiological studies on adverse dieting behaviours and eating disorders among young people in Hungary". Social Psychiatry and Psychiatric Epidemiology:39 (8): 647-54.

Tremblay, Mark Stephen; Colley, Rachel Christine; Saunders, Travis John; Healy, Genevieve Nissa; Owen, Neville (2010). "Physiological and health implications of a sedentary lifestyle". Applied Physiology, Nutrition, and Metabolism:35 (6): 725-740.

Vogel, M., Schwarze-Zander, C., Wasmuth, J. C., Spengler, U., Sauerbruch, T. and Rockstroh, J. K. (2010). "The treatment of patients with HIV" DeutschesÄrzteblatt international107 (2829): 507-15; quiz 516.

Walker, B. D. (2007). "Elite control of HIV Infection: implications for vaccines and treatment". Topics in HIV medicine: a publication of the International AIDS Society USA:15 (4): 1346.

Walsh, J. M., Wheat, M. E., Freund, K. and Wheat, F. (2000). "Detection, evaluation, and treatment of eating disorders". Journal of General Internal Medicine: 15 (8): 577-90.

Weiss, S. (1995). A comparison of maladaptive behaviors of athletes and non-athletes. The Journal of Psychology: 133(3), 315-322.

Westerburg, D. P. and Waitz, M. (2013). "Binge-eating disorder". Osteopathic Family Physician:5 (6): 230-33.

What are Eating Disorders?NIMH (2015).

Whitnet, E. and Rolfes, S. R. (2011).Understanding Nutrition. United States: Wadsworth Cengage Learning. p. 255. ISBN 1-133-58752-6.

Winslow, C. E. (1920). "The Untilled Fields of Public Health". Science:51 (1306): 23-33.

Workowski, K. A. and Bolan, G. A. (2015). "Sexually transmitted diseases treatment guidelines, 2015." MMWR-Recommendations and reports: Morbidity and mortality weekly report. Recommendations and reports Centers for Disease Control64 (RR-03): 1-137.

World Health Organization, (2013). Cardiovascular diseases (CVDs) Fact sheet no. 317 [Online]. World Health Organization (Available: 
(Online)

http://www.who.int/mediacentre/factsheets/fs317/en/).

World Health Organization (2011). Global recommendations on: Health. Geneva: World Health Organization.

Word Health Organization, (2011). Global Status Report on Alcohol and Health. Geneva: World Health Organization.

World Health Organization's (2010). global recommendations on: Physical Activity for Health. Geneva: World Health Organization.

World Health Organization BMI Classification (2006).Global Database on Body Mass Index

World Health Organization (2003). "Gender, Health and Ageing “(PDF).

World Health Organization (2002).Reducing risks,promoting healthy life. World Health Report 2002 Geneva, Switzerland. WHO Press

World Health Organization (1998). List of Basic Terms. Health Promotion Glossary: (pp. 4)

World Health Organization (1946). Preamble to the Constitution of the World Health Organization as adopted by the International Health Conference, New York, signed by the representatives of 61 States (Official Records of the World Health Organization, no. 2, p. 100) and entered into force in 1948.

Yates, B., \& White, S. (2004). Incidence and risk factors in the development of medial tibia stress syndrome among naval recruits. The American Journal of Sports Medicine; 32.3: 772-780

Zimmer, B. (2010)."Wellness"The New York Times.

Conflict of Interest: None declared 